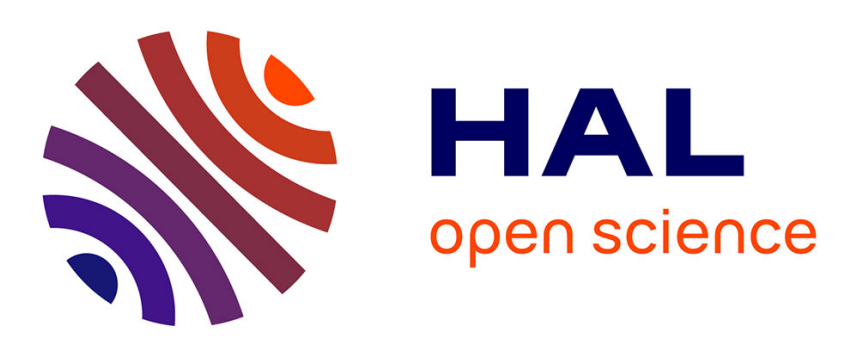

\title{
Entity Local Structure Graph Matching for Mislabeling Correction
}

Nihel Kooli, Abdel Belaïd, Aurélie Joseph, Vincent Poulain D 'Andecy

\section{To cite this version:}

Nihel Kooli, Abdel Belaïd, Aurélie Joseph, Vincent Poulain D 'Andecy. Entity Local Structure Graph Matching for Mislabeling Correction. Document Analysis Systems, Apr 2016, Santorini, Greece. pp.257-262, 10.1109/DAS.2016.36 . hal-01304257

\section{HAL Id: hal-01304257 \\ https://inria.hal.science/hal-01304257}

Submitted on 21 Jul 2016

HAL is a multi-disciplinary open access archive for the deposit and dissemination of scientific research documents, whether they are published or not. The documents may come from teaching and research institutions in France or abroad, or from public or private research centers.
L'archive ouverte pluridisciplinaire HAL, est destinée au dépôt et à la diffusion de documents scientifiques de niveau recherche, publiés ou non, émanant des établissements d'enseignement et de recherche français ou étrangers, des laboratoires publics ou privés. 


\section{Entity Local Structure Graph Matching for Mislabeling Correction}

\author{
Nihel Kooli and Abdel Belaïd \\ LORIA - Université de Lorraine \\ Campus scientifique - BP 239 \\ 54506 Vandoeuvre-lès-Nancy, France \\ Email: \{nihel.kooli,abdel.belaid\}@loria.fr
}

\author{
Aurélie Joseph and Vincent Poulain D'Andecy \\ ITESOFT Groupe - YOOZ \\ Parc d'Andron, Le Séquoïa \\ 30470 Aimargues, France \\ Email: \{aurelie.joseph,vincent.poulaindandecy\}@yooz.fr
}

\begin{abstract}
This paper proposes an entity local structure comparison approach based on inexact subgraph matching. The comparison results are used for mislabeling correction in the local structure. The latter represents a set of entity attribute labels which are physically close in a document image. It is modeled by an attributed graph describing the content and presentation features of the labels by the nodes and the geometrical features by the arcs. A local structure graph is matched with a structure model which represents a set of local structure model graphs. The structure model is initially built using a set of well chosen local structures based on a graph clustering algorithm and is then incrementally updated. The subgraph matching adopts a specific cost function that integrates the feature dissimilarities. The matched model graph is used to extract the missed labels, prune the extraneous ones and correct the erroneous label fields in the local structure. The evaluation of the structure comparison approach on 525 local structures extracted from 200 business documents achieves about $90 \%$ for recall and $95 \%$ for precision. The mislabeling correction rates in these local structures vary between $73 \%$ and $100 \%$.
\end{abstract}

Keywords-entity local structure; subgraph matching; mislabeling correction; structure model; graph clustering;

\section{INTRODUCTION}

An entity is defined as an existing or real thing, such as a person, a location, an organization, etc. It is contrasted with its attributes. For example, a person has the attributes: name, date of birth, social security number, etc. Extracting entities in documents is equivalent to identifying their attributes. This work lies in the context of entity extraction in document images guided by a predefined database, as reported in [1]. This database describes the entity attributes by its records and informs about their semantics by its fields (columns). The entity attributes are labeled in the document, as proposed in our earlier work [2], using dictionaries extracted from noun fields in the database and regular expression built from fields having a standard from. However, mislabeling may frequently occur as missed labels, extraneous labels or erroneous label fields. The impact is a final wrong detection of an entity. For example, if the social security number is wrongly detected, the person may be not detected or worst confused in the database with an other person having the same name. Missed labels are caused by OCR errors or non-standardized representations between the document and the database (for example, term permutation, abbreviations, typing errors, etc.). Erroneous labels are caused by a confusing syntax of some attributes, for example an amount number labeled by mistake as a zip-code. Finally, the extraneous labels represent noise.

In this paper, we propose to correct the mislabeling by employing the entity label arrangement in the document. We assume that attributes (labels within the page) which are physically close in the document page are more probably related to the same entity than the same ones faraway. These labels build a local structure which is modeled by a labeled graph, called entity local structure graph. In this graph, the nodes represent the content features (syntactic and semantic) and the presentation features of the labels and the arcs represent the geometric relationships between them. This graph is matched with a structure model, which is composed of a set of entity local structure graphs.

Labeled graphs are data structures that offer a significant modeling of complex entities. The labeled nodes describe the entity elements and the labeled arcs represent the relationships between them. Some previous works propose to employ graph modeling for semantic labeling in document images. For example, authors in [3] and [4] propose to model the document layout by graphs for logical labeling and document classification in journals and conference proceedings. This work is based on a model which is learned for each document class. The model represents spatial relationships between the blocks segmented by the OCR. However, this approach is dependent on the document class. The same authors propose in [5] to add the document content study to its layout modeling in order to enhance the labeling in English business letters. However, this approach does not deal with the noisy content given by the OCR.

Labeled graph matching is usually an NP-complete problem and whose objective is to find a way to correspond a graph to another, such that the topology and the node and arc labels are matched. In the domain of pattern recognition, graph matching techniques are generally used to correspond between an observed graph, called candidate graph and a known entity graph, called model graph. There exist two types of graph matching techniques [6] [7] in the literature: exact graph matching and inexact graph matching. Exact graph matching is to find an isomorphism between graphs. Inexact graph matching is the most commonly used and consists on searching for the best match by tolerating the noisy representation and the errors. For this purpose, the most used approach is the Graph Edit Distance (GED) [8]. GED is inspired from the string edit distance and is defined as the weighted sum of the 
edit operation costs which are needed to transform one graph to the other. However, in the case of labeled graph where the labels are not of nominal type but represent a set of numeral attributes resulting from a feature extraction step, it is generally not possible to find an exact mapping between these labels. To deal with such a problem, a label discretization based solution can be proposed to transform the numerical attributes into nominal labels, but it is clear that the mapping is sensitive to discretization errors. Another solution consists of defining the mapping cost as the sum of distances between the label values and using a cost threshold for the label mapping decision, but it is not easy to find the proper one. This problem was treated in [9] by reformulating the problem in the Integer Linear Program (ILP) formalism and integrating the label mapping cost in the subgraph matching cost. However, this approach tolerates only the label substitution operation and does not deal with the deletion of nodes or arcs.

Our graph matching strategy lies with inexact subgraph matching which consists on searching an inexact matching between a candidate graph and a subgraph of a model graph. This paper treats also labeled graphs where the labels represent a set of numerical attributes (scalar or vectorial). The graph matching problem is solved by the integration of the feature dissimilarities in the cost function. This work is a thorough extension of the work reported in [2] which performs substitution tolerant subgraph matching for the entity structure correction and involves human for the structure model learning. This paper deals with the node and arc deletion in the subgraph matching in order to tolerate the noisy representation of real graphs and extends the label representation features in the local structures. Furthermore, it proposes an unsupervised learning of the structure model based on a graph clustering algorithm.

The remainder of this paper is organized as follows. The entity structure matching approach is detailed in Section II. The experiments on real world data are presented and analyzed in Section III. The paper is concluded in Section IV.

\section{ENTITY STRUCTURE MATCHING APPROACH}

Fig. 1 presents the global schema of the proposed approach. Firstly, a candidate graph is built for each local structure extracted from an input document. Secondly, the candidate graph is compared to the structure model using a subgraph matching method. This model is initially learned from a chosen dataset using a graph clustering algorithm and is then incrementally updated for a document stream. Finally, the geometrical relations in the matched model graph are used to correct the eventual mislabeling in the local structure.

\section{A. Local structure representation}

A label in the local structure is defined as:

$$
l_{i}=\left(c_{i}, v_{i}, \operatorname{con} f_{i}\right)
$$

where $v_{i}$ is the value of the label, $c_{i}$ is the corresponding field (column) in the database and conf $f_{i}$ is the confidence of labeling $l_{i}$ given by the n-gram distances [10] which is used to compare between string values in the dictionary and in the document. $v_{i}$ is represented by a bag of words $v_{i}=\left\{t_{j}\right\}$. Each input document is defined by $d=\left\{l_{i}\right\}$.

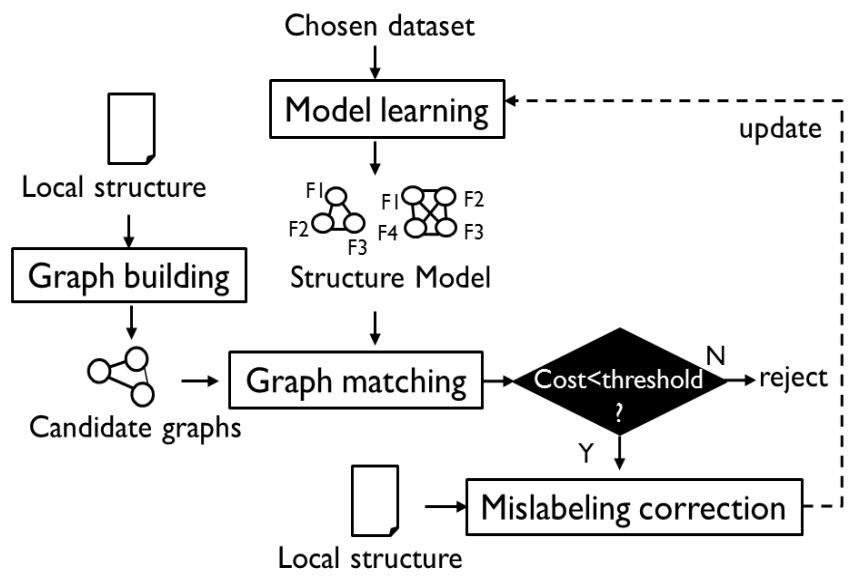

Fig. 1. Global schema of the proposed system

An entity local structure in the document is modeled by a directed complete graph $G=(N, A, \mu, \xi)$ where $N$ is a finite set of nodes that correspond to semantic labels, $A \subseteq$ $N \times N$ is a finite set of arcs that represent geometrical relations between the nodes, $\mu: N \rightarrow L_{N}$ and $\xi: A \rightarrow L_{A}$ are two functions assigning a label to a node and an arc respectively. $L_{N}$ and $L_{A}$ are discrete sets of labels for the nodes and the arcs respectively. Each arc $a_{i j} \in A$ relating the nodes $n_{i}$ and $n_{j}$ is represented by $n_{i} n_{j}$. A node $n_{i}$ that corresponds to a label $l_{i}$ is defined by:

$$
n_{i}=\left(c_{i}, \operatorname{conf}_{i}, n t_{i}, n l_{i}, p_{i}\right)
$$

where $n t_{i}$ is the number of label terms, $n l_{i}$ is the number of label lines and $p_{i}$ is the normalized font size according to the average font of the document (small: 0 , medium: $\frac{1}{2}$, large: 1 ). For an arc $a_{i j}$ relating the nodes $n_{i}$ and $n_{j}$, we define a feature vector describing the spatial relation between these nodes as:

$$
a_{i j}=\left(v s_{i j}, h s_{i j}, a l_{i j}\right)
$$

where vs (vertical separation) is the number of lines that separate the labels corresponding to $n_{i}$ and $n_{j}$. hs (horizontal separation) is the distance, in number of characters, that separates the bounding boxes of the labels corresponding to $n_{i}$ and $n_{j} . v s_{i j}$ and $h s_{i j}$ are signed to inform about the relative vertical position (above, below) or the relative direction (on the right, on the left). $a l_{i j}=\left(\right.$ rJust $_{i j},{ }_{\text {lJ }}$ sst $_{i j}$, cent $\left._{i j}\right)$ is a vector of three binary values which inform about line alignment (right aligned, left aligned, centered text). Slight variation (lower than 20 pixels) between the line boundaries is tolerated for the alignment.

Fig. 2 shows examples of local structures extracted from real world document images with their representations by attributed graphs (for simplicity, we do not represent all the arcs in the graphs). Fig. 2 (a) and Fig. 2 (b) are extracted from administrative documents. They model an address and a fiscal information structures respectively. Fig. 2 (c) represents a bibliography header structure extracted from a scientific paper.

\section{B. Structure comparison}

This can be formulated as a problem of inexact subgraph matching between a candidate graph and a model graph in 


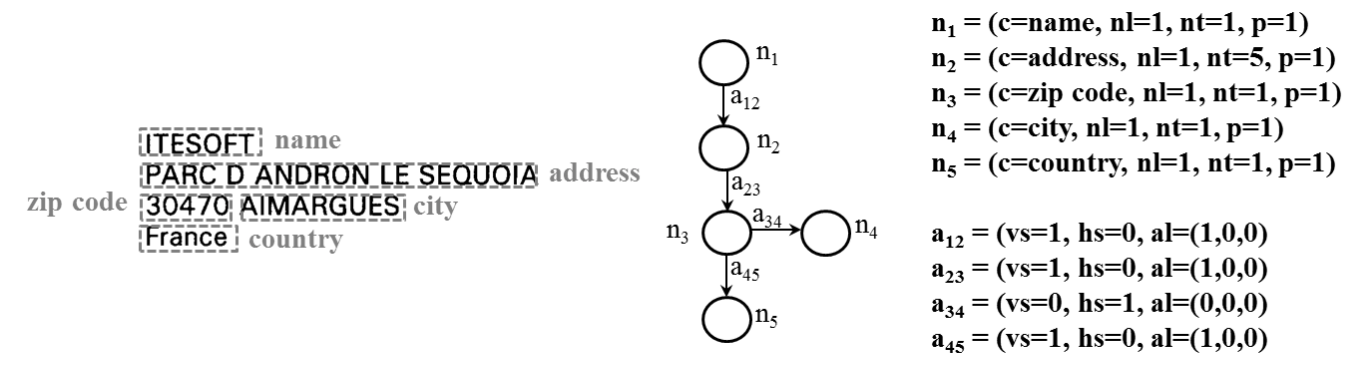

(a) an address structure

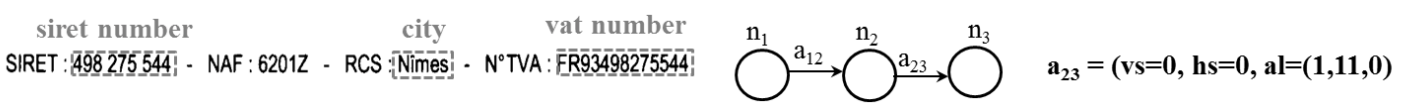

(b) a fiscal information structure
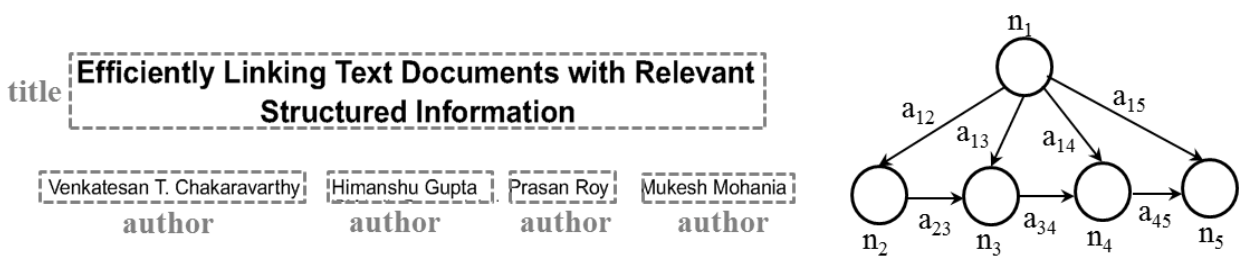

(c) a bibliography reference header structure

Fig. 2. Examples of local structures

the structure model. The following distortions are considered: arc and node label distortions (variation in their attributes) and extraneous arcs or nodes in the candidate graph. It is not necessary to deal with missing arcs or nodes since we search for a subgraph matching between a candidate graph and a model graph. In order to solve this problem which is generally NP-hard, we use the Branch and bound algorithm [11] which proposes a tree search of the node mapping with backtracking using heuristics. This algorithm is easily adapted to our context since it takes into account the node and arc attributes to provide the matching heuristics. In fact, we employ semantic heuristics (for example, a node labeled by a zip code can not be mapped to a node labeled by a name in an address structure) and physical heuristics (for example, a node labeled by an author can not be placed above a node labeled by a title in a bibliography reference header structure).

Let $t: G \rightarrow M \cup\{\epsilon\}$ be a graph mapping function from a candidate graph $G=\left(N_{G}, A_{G}, \mu_{G}, \xi_{G}\right)$ to a subgraph of a model graph $M=\left(N_{M}, A_{M}, \mu_{M}, \xi_{M}\right)$. To allow the node deletion, it is possible to map a node in $G$ to $\epsilon$. The graph matching cost for the mapping $t$ from $G$ to $M$ is defined as:

$$
\begin{aligned}
C(G, M, t)=\frac{\alpha}{\left|N_{G}\right|} \sum_{n \in N_{G}} C_{N}(n, t(n))+ & \\
& \frac{1-\alpha}{\left|A_{G}\right|} \sum_{n \in G} \sum_{n^{\prime} \in G} C_{A}\left(n n^{\prime}, t(n) t\left(n^{\prime}\right)\right)
\end{aligned}
$$

where $\alpha \in[0,1]$ and $C_{N}: N_{G} \times N_{M} \rightarrow R^{+}$and $C_{A}$ : $A_{G} \times A_{M} \rightarrow R^{+}$are the mapping cost functions for the nodes and the arcs respectively. Let $\Delta$ be the set of the possible mapping functions from $G$ to a subgraph of $M$. The matching cost is then defined as:

$$
C(G, M)=\min _{t \in \Delta} C(G, M, t)
$$

Let $F_{N}=\{n t, n l, p\}$ and $F_{A}=\{v s, h s, a l\}$ be the sets of node and arc features respectively. The node mapping cost function from $n=(c$, conf, $n t, n l, p)$ to $n^{\prime}=$ $\left(c^{\prime}\right.$, conf $\left.^{\prime}, n t^{\prime}, n l^{\prime}, p^{\prime}\right)$ is then defined as:

$$
C_{N}\left(n, n^{\prime}\right)= \begin{cases}\lambda_{n^{\prime}}\left(1-\text { conf.conf } f^{\prime}\right) & \text { if } c=c^{\prime} ; \\ \lambda_{n^{\prime}} \sum_{f \in F_{N}} \lambda_{f} d_{f}\left(n, n^{\prime}\right) & \text { else. }\end{cases}
$$

The arc mapping cost function from $a=(v s, h s, a l)$ to $a^{\prime}=$ $\left(v s^{\prime}, h s^{\prime}, a l^{\prime}\right)$ is defined as:

$$
C_{A}\left(a, a^{\prime}\right)=\lambda_{a^{\prime}} \sum_{f \in F_{A}} \lambda_{f} d_{f}\left(a, a^{\prime}\right)
$$

where $\lambda_{n^{\prime}}, \lambda_{a^{\prime}} \in[0,1]$ are the weight factors for nodes and arcs in the model graph and $\lambda_{f} \in[0,1]$ depends on the feature relevance. Dissimilarities of scalar features are defined as:

$$
d_{f}\left(a, a^{\prime}\right)=\left|f^{N}-f^{\prime N}\right| \quad \forall f \in F_{N} \cup F_{A} \backslash\{a l\}
$$

$f^{N}$ is the normalized value of $f$ defined by:

$$
f^{N}=\frac{f-\max (f)}{\max (f)-\min (f)}
$$

where $\max (f)$ and $\min (f)$ represent the maximum and the minimum values respectively and are dataset dependent.

The alignment dissimilarity is defined as:

$$
d_{a l}\left(a, a^{\prime}\right)= \begin{cases}0 & \text { if } a l \times a l^{\prime} \neq 0 \\ 1 & \text { else }\end{cases}
$$

Comparing a candidate graph to all model graphs in the structure model is time consuming. We propose then to 
filter these graphs using semantic heuristics (for example, the number of nodes having a common label field) and structural heuristics (for example, the number of lines in the local structure or the logical order of the labels in a page line or column). Given a model graph dataset $S_{M}=\left\{M_{1}, \ldots, M_{n}\right\}$ and one candidate graph $G$, the structure comparison is equivalent to the selection of the model graph $M_{\text {match }}$, where:

$$
M_{\text {match }}=\arg \min _{M_{i} \in S_{M}} C\left(G, M_{i}\right)
$$

The model $M_{\text {match }}$ is retained if the matching cost does not exceed an empirically fixed threshold.

\section{Mislabeling correction}

A matched model graph with a candidate is used to correct the three types of mislabeling (missed labels, erroneous label fields and extraneous labels in the local structure modeled by this candidate graph). The extraneous nodes in the model graph may correspond to missed labels in the local structure. The geometrical relations provided by the arcs related to these extraneous nodes are used to localize the missed labels in the document. To validate these label correspondence to the candidate entity in the database, their values are compared to the entity attributes by being less strict in the string distances. Furthermore, the substituted node labels in the model graph are used to correct the label fields in the local structure. Finally, the labels corresponding to deleted nodes in the candidate graph are pruned in the local structure.

Fig. 3 shows an example of mislabeling correction in a structure model. In Fig. 3 (a), the value "3091Z" was labeled as a zip code due to a confusion made by the OCR of the character " $Z$ " with the character " 7 ". Furthermore, the mail was not labeled due to OCR errors and the fax was labeled as a phone since they have the same syntax. The candidate graph built from this local structure is shown in Fig. 3 (b). The matched model graph with this candidate graph using inexact subgraph matching is shown in Fig. 3 (c). This model graph is used to extract the missed mail, to correct the erroneous phone and to prune the extraneous zip code in the local structure represented in Fig. 3 (a).

\section{Structure model learning}

The structure model is initially learned via the incremental graph clustering algorithm detailed in Algorithm 1. The latter is executed on a dataset chosen to be representative of the corpus. It is an improvement of the Leaders algorithm [12] by adding the incremental update of the centroid to be more representative of the cluster members. This algorithm is adapted to our case since it is a simple incremental clustering algorithm that requires one dataset scan. Besides, experiments on our corpus show that the performances are not affected by the data stream order. During the document stream processing, the structure model is incrementally updated. In fact, each model graph matched with a treated candidate graph is recomputed by considering this new candidate graph.

Let us define now the distance and the centroid mentioned in the algorithm: the distance is the graph matching cost function and the centroid is the group representative graph. The representative graph structure is built using the concept

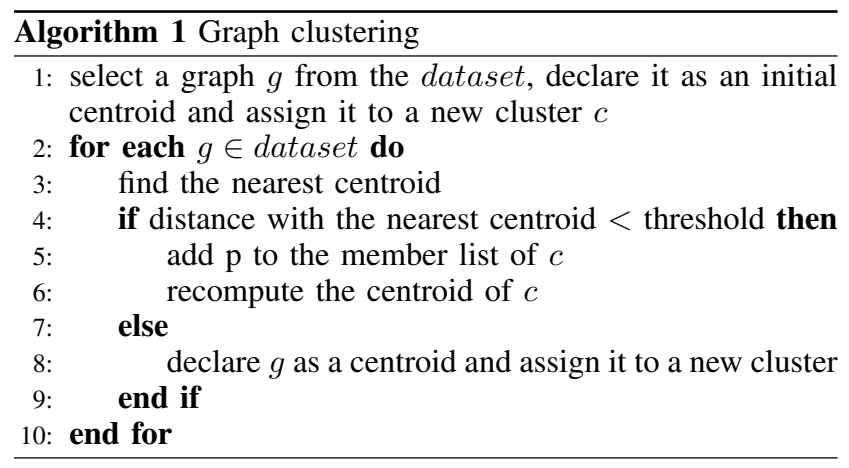

of "Weighted Minimum Common Supergraph (WMCS)" proposed in [13]. The representative graph attributes are learned by fusing the attributes of the member graphs in the cluster. For distances, the sample average is computed. For alignment, the dominant value is used. The representative graph weight factors are set up inversely proportional to the deviation of the attributes in the samples. That is to say, the larger the sample variation of an attribute is, the less discriminant it is and so the lower its weight factor is.

\section{EXPERIMENTS}

We work on a real-world industrial project in direct collaboration with the ITESOFT ${ }^{1}$ company. For tests, we use a dataset of 525 local structures extracted from 200 printed documents. These documents represent industrial invoices or purchase orders. The local structures are categorized into 4 types: postal address structures, contact information structures, fiscal information structures and mixed structures which are a combination of two or more types of structures. For the entity attribute labeling in the documents, a company intern tool called FullText is used. The dictionaries and the regular expressions are built from a database which is composed of a table containing about 230000 records, where each one denotes an entity. This database registers information about enterprises (industrial suppliers and clients) such as their names, addresses, contact numbers, etc.

First, we evaluate the local structure graph matching approach on our corpus. Then, we highlight its benefits for the mislabeling correction. Finally, we evaluate the model learning module based on the graph clustering algorithm.

\section{A. Graph matching results}

For the graph matching experiments, we use a structure model composed of 41 model graphs (the experiments on the model learning will be presented in section III-C) and a dataset of 525 graph candidates built from the local structure samples. For evaluation, we use a ground truth table that links each candidate graph with its model graph. This ground truth was manually prepared by an expert.

A relevant model graph for a candidate graph is defined as a graph that contains a subgraph corresponding to this candidate graph. The precision and recall are then defined as:

$$
\text { recall }=\frac{\# R M G}{\# R G} \quad \text { precision }=\frac{\# R M G}{\# M G}
$$

${ }^{1}$ http://www.itesoft.com 


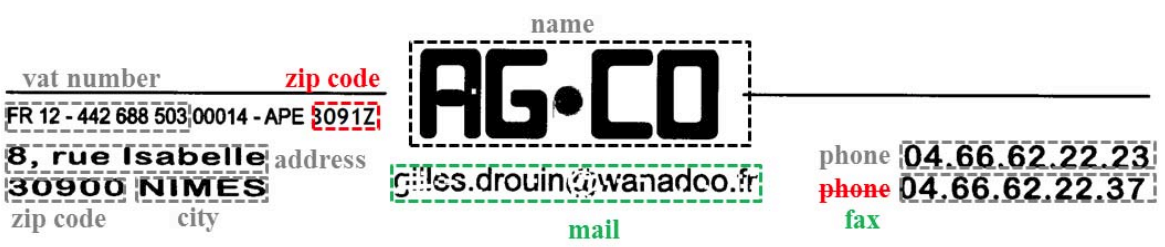

(a) An example of local structure with mislabeling

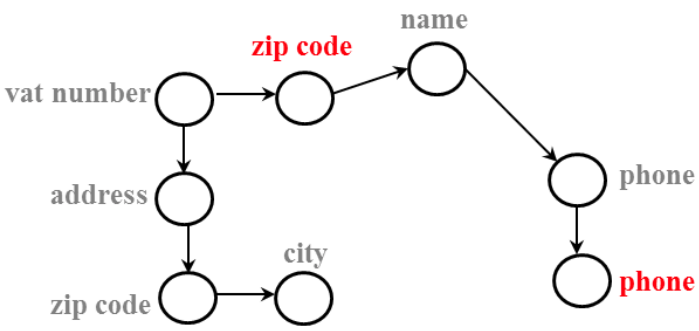

(b) The corresponding candidate graph

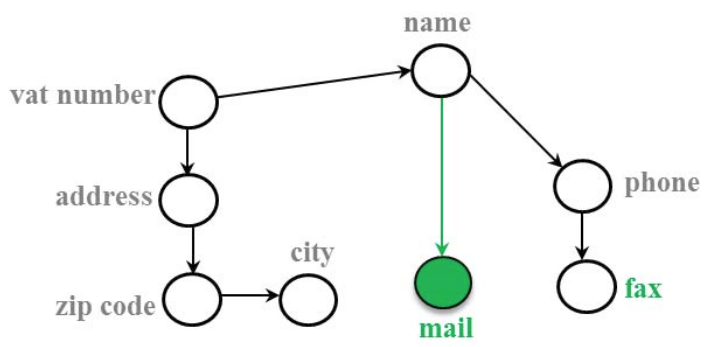

(c) The matched model graph

Fig. 3. Mislabeling correction in a local structure using subgraph matching

where $R G, M G$ and $R M G$ represent the relevant graphs, the matched graphs and the relevant matched graphs respectively.

Two strategies are used for the evaluation: always match a candidate graph with the best model graph (top 1) or match it with the best model graph only if their matching cost is below a threshold. The results are illustrated in Table I. It shows that the graph matching algorithm performs well for all structure types. Besides, it shows that the acceptance threshold strategy gives better precision (95.78\% compared to $92.57 \%$ ) and f-measure (93.26\% compared to $92.57 \%$ ) than the best match strategy even if the recall ( $90.86 \%$ compared to $92.57 \%$ ) is worse. This is explicated by the considerable reduction of the false positives due to the rejection by the threshold. In order to limit the wrong corrections of the local structures, the acceptance threshold strategy is adopted for the remaining experiments.

The graph matching method is compared with existent methods in Table II. Results show that our method outperforms the GED + label discretization and the GED + decision threshold ones using either the substitution tolerant subgraph matching or the substitution and deletion tolerant subgraph matching due to the integration of the feature dissimilarities in the matching cost function. Furthermore, it gives better precision but worse recall than the optimization based method in the case of substitution tolerant subgraph matching. This is explained by the employed heuristics, in the branch and bound algorithm, which discard some "wrong" matching solutions.

\section{B. Mislabeling correction results}

The three types of mislabeling correction, using the structure matching, are evaluated separately on a set of labels of variant fields. Missed labels detection in the local structures is evaluated on a set of missed labels using a manually prepared ground truth. TABLE III presents the obtained results. It shows that the identification of all column labels gets high recall (varied between $73.75 \%$ and $96 \%$ ) and precision (varied between $84.28 \%$ and $100 \%$ ) rates. The erroneous label fields correction and the extraneous labels pruning are similarly evaluated and results are presented in TABLE IV and TABLE $\mathrm{V}$ respectively. Results are promising for both recall and precision rates. In fact, the recall (precision) rates are varied between $75 \%$ and $96 \%$ (90.91\% and $100 \%)$ for erroneous label fields correction and between $77.14 \%$ and $97.5 \%(91.53 \%$ and $100 \%$ ) for extraneous labels pruning. Errors cases are essentially caused by a wrong graph matching solution or a deficiency in verifying the similarity between the detected missed label value and the entity attribute value in the database.

\section{Model learning results}

For the clustering, a dataset of 290 local structure graphs extracted from 87 documents is used. The number of obtained clusters (model graphs) is 41 . To evaluate the clustering algorithm, the Dunn index $D$ is used. It is defined as:

$$
D=\frac{\min _{1 \leq i<j \leq n} d(i, j)}{\max _{1 \leq k \leq n} d^{\prime}(k)}
$$

where $d(i, j)$ is the inter-cluster distance between the clusters $i$ and $j$ (the distance between the centroids) and $d(k)$ is the intra-cluster distance of the cluster $k$ (the maximal distance between any element pair in the cluster $k$ ).

The Dunn index is evaluated for three variant stream orders of processed graphs in the clustering algorithm. The obtained values are around 0.74 for the three stream orders with the same number of obtained clusters (41 clusters). These results prove that our corpus is independent of the data stream order.

Table VI presents a comparison of the clustering results between the proposed algorithm and the Leaders one. Its shows that the proposed algorithm outperforms the Leaders one with a Dunn index of 0.74 compared to 0.71 . This justifies our choice consisting of the incremental update of each cluster centroid. 
TABLE I. STRUCTURE GRAPH MATCHING RESULTS

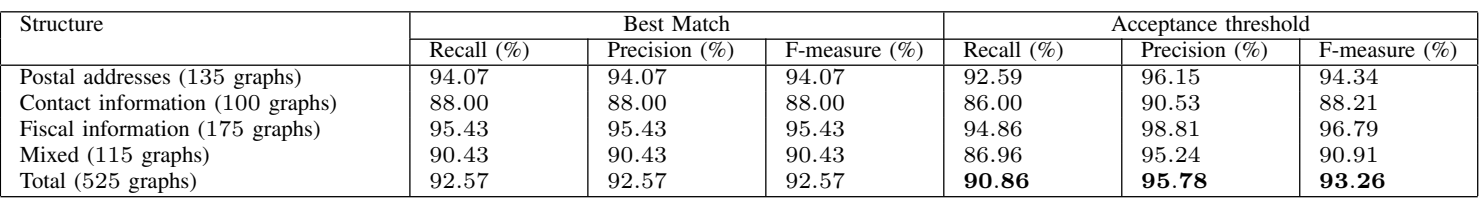

TABLE II. GRAPH MATCHING COMPARISON RESULTS

\begin{tabular}{|l|l|l|l|l|}
\hline & Method & Recall (\%) & Precision (\%) & F-measure (\%) \\
\hline \multirow{3}{*}{ Substitution tolerant subraph matching } & GED + Label discretization & 86.10 & 94.76 & 90.22 \\
& GED + Decision threshold & 86.67 & 95.39 & 90.82 \\
& Optimizaton [9] & 88.00 & 95.45 & 91.58 \\
& Our earlier work [2] & 87.81 & 96.04 & 91.74 \\
\hline \multirow{2}{*}{ Substitution and deletion tolerant subraph } & GED + Label discretization & 87.81 & 94.66 & 91.11 \\
matching & GED + Decision threshold & 88.57 & 94.90 & 91.63 \\
& Our method & $\mathbf{9 0 . 8 6}$ & $\mathbf{9 5 . 7 8}$ \\
\hline
\end{tabular}

TABLE III. MISSED LABEL IDENTIFICATION RATES

\begin{tabular}{|l|l|l|l|l|l|}
\hline \multirow{2}{*}{ Label field } & \multicolumn{3}{|c|}{ Number of labels } & Recall (\%) & Precision (\%) \\
\cline { 2 - 4 } & Missed & Found & Correct & & \\
\hline Name & 80 & 70 & 59 & 73.75 & 84.28 \\
Address & 80 & 73 & 65 & 81.25 & 89.04 \\
Zip code & 100 & 96 & 96 & 96 & 100 \\
City & 100 & 91 & 89 & 89 & 97.80 \\
Country & 50 & 45 & 41 & 82 & 91.11 \\
Phone & 70 & 62 & 60 & 85.71 & 96.77 \\
Fax & 70 & 60 & 60 & 85.71 & 100 \\
mail & 50 & 44 & 44 & 88 & 100 \\
Vat number & 50 & 42 & 39 & 78 & 92.86 \\
Siret number & 50 & 41 & 37 & 74 & 90.24 \\
\hline
\end{tabular}

TABLE IV. ERRONEOUS LABEL FIELDS CORRECTION RATES

\begin{tabular}{|l|l|l|l|l|l|}
\hline \multirow{2}{*}{ Label field } & \multicolumn{3}{|c|}{ Number of labels } & Recall (\%) & Precision (\%) \\
\cline { 2 - 4 } & Erroneous & Substituted & Correct & & \\
\hline Zip code & 100 & 96 & 96 & 96 & 100 \\
Name & 100 & 81 & 75 & 75 & 92.59 \\
City & 100 & 91 & 88 & 88 & 96.70 \\
Phone & 50 & 44 & 40 & 80 & 90.91 \\
Fax & 50 & 47 & 45 & 90 & 95.74 \\
\hline
\end{tabular}

TABLE V. EXTRANEOUS LABELS PRUNING RATES

\begin{tabular}{|l|l|l|l|l|l|}
\hline \multirow{2}{*}{ Label field } & \multicolumn{3}{|c|}{ Number of labels } & Recall (\%) & Precision (\%) \\
\cline { 2 - 4 } & Extraneous & Pruned & Correct & & \\
\hline Zip code & 80 & 78 & 78 & 97.5 & 100 \\
Name & 100 & 82 & 78 & 78 & 95.12 \\
City & 100 & 87 & 85 & 85 & 97.70 \\
Phone & 70 & 59 & 54 & 77.14 & 91.53 \\
Siret number & 50 & 46 & 45 & 90 & 97.83 \\
\hline
\end{tabular}

TABLE VI. CLUSTERING COMPARISON RESULTS

\begin{tabular}{|l|l|l|l|}
\hline Method & Best threshold & \# Cluster & Dunn index \\
\hline Leaders [12] & 0.1 & 42 & 0.71 \\
Our method & $\mathbf{0 . 1}$ & $\mathbf{4 1}$ & $\mathbf{0 . 7 4}$ \\
\hline
\end{tabular}

\section{CONCLUSION AND FUTURE WORK}

This paper proposes a structure comparison approach for mislabeling correction in documents using a structure model. The comparison is based on a subgraph matching method between a candidate graph (built for each local structure) and a model graph (in the structure model). The evaluation on a dataset of 525 local structures extracted from 200 documents is promising and achieves about $90 \%$ for recall and $95 \%$ for precision. Furthermore, the method has proven to be effective in correcting the mislabeling (missed labels, erroneous label fields and extraneous labels) in the local structures.

Our future work is to evaluate our approach on other datasets in order to investigate more local structure types such as table lines and bibliographic references. Another interesting work is to enhance the verification of the identified missed labels by combining different similarity measures and using an OCR correction model based on character shape classification Furthermore, we plan to propose a probabilistic score of entity matching in document images based on an estimation of the belonging of their labeled attributes to the local structures.

\section{REFERENCES}

[1] N. Kooli and A. Belaïd, "Entity Matching in OCRed Documents with Structured Databases", in International Conference on Pattern Recognition Applications and Methods, 2015, pp. 165-172.

[2] N. Kooli and A. Belaïd, "Semantic Label and Structure Model based Approach for Entity Recognition in Database Context", in International Conference on Document Analysis and Recognition, 2015, pp. 301-305.

[3] J. Liang and D. S. Doermann, "Logical labeling of document images using layout graph matching with adaptive learning", in Document Analysis System, 2002, pp. 224-235.

[4] J. Liang, D. S. Doermann, M. Y. Ma and J. K. Guo, "Page Classification through Logical Labelling", in International Conference on Pattern Recognition, 2002, pp. 477-480.

[5] J. Liang and D. S. Doermann, "Content features for logical document labeling", in Document Recognition and Retrieval, 2003, pp. 189-196.

[6] D. Conte, P. Foggia, C. Sansone and M. Vento, "Thirty years of graph matching in pattern recognition", International Journal of Pattern Recognition and Artificial Intelligence, 2004, 18(3): pp. 265-298.

[7] P. Foggia, G. Percannella and M. Vento, "Graph Matching and Learning in Pattern Recognition in the last 10 years", International Journal of Pattern Recognition and Artificial Intelligence, 2014, 28(1): pp. 14500011-1450001-40.

[8] A. Sanfeliu, KS. Fu, "A distance measure between attributed relational graphs for pattern recognition", IEEE Transactions on Systems Man and Cybernetics, 1983, 13(3): pp. 353-362.

[9] P. Le Bodic, P. Hroux, S. Adam and Y. Lecourtier, "An Integer Linear Program for Substitution Tolerant Subgraph Isomorphism and its Use for Symbol Spotting in Technical Drawings", Pattern Recognition, 2012, 45(12): pp. 4214-4224.

[10] G. Kondrak, "N-Gram Similarity and Distance", in International conference on String Processing and Information Retrieval, 2005, pp. 115-126.

[11] A. K. C. Wong, M. You and S. C. Chan, "An algorithm for graph optimal monomorphism", IEEE Transactions on System Man and Cybernetics, 1990, 20(3): pp. 628-638.

[12] P.A. Vijaya, M. Narasimha Murty and D.K. Subramanian, "LeadersSubleaders: An efficient hierarchical clustering algorithm for large data sets", in Pattern Recognition Letters, 2004, 25(4): pp. 505-513.

[13] H. Bunke, P. Foggia, C. Guidobaldi and M. Vento, Graph clustering using the weighted minimum common supergraph, in International Conference on Graph based Representations in Pattern Recognition, 2003 , pp. 235-246. 